10

SSCL-Preprint-510

October 1993

Distribution Category: 414

J. Ellison

H.-J. Shih

M. Kummer

Theoretical Study of Longitudinal Beam Splitting and Related Phenomena

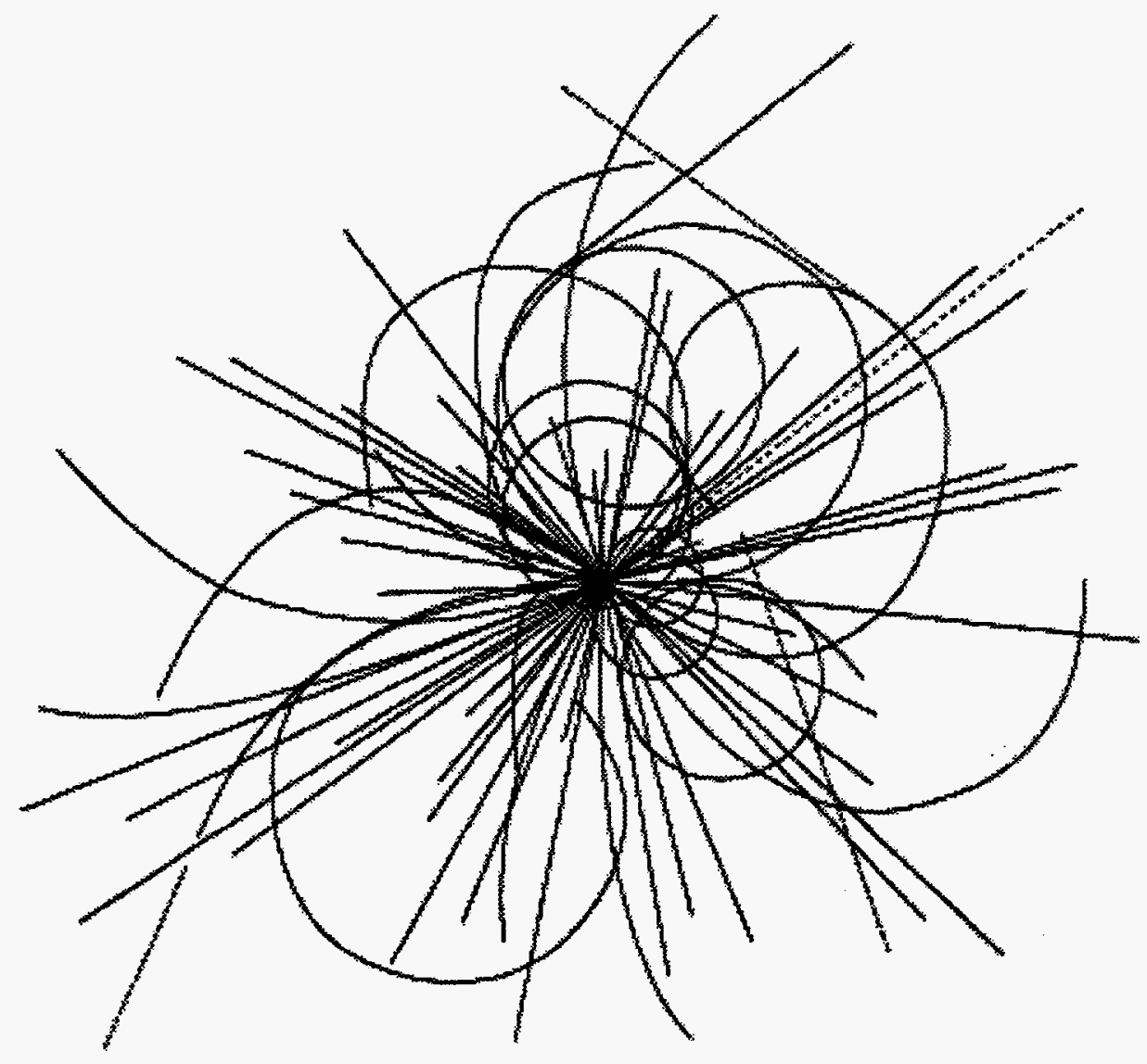

\section{Superconducting Super Collider Laboratory}

APPRONED FOR RELEASE OR

PUBLSOION - OR.PATENT GROUP BY. E. ........ DATE 


\section{Disclaimer Notice}

This report was prepared as an account of work sponsored by an agency of the Untted States Government. Neither the United States Government or any agency thereot, nor any of their employees, makes any warranty, express or implied, or assumes any legal ilability or responsibility for the accuracy, completeness, or usefuness of any information, apparatus, product, or process disclosed, or represents that its use would not infringe privately owned rights. Reference herein to any specific commercial product, process, or service by trade name, trademark, manufacturer, or otherwise, does not necessarity constitute or imply its endorsement, recommendation, or favoring by the United States Government or any agency thereof. The views and opinions of authors expressed herein do not necessarily state or reflect those of the United States Govemment or any agency thereof.

Superconducting Super Collider Laboratory is an equal opportunity employer. 
SSCL-Preprint-510

\title{
Theoretical Study of Longitudinal Beam Splitting and Related Phenomena*
}

\author{
J. Ellison ${ }^{\dagger}$ and H.-J. Shih \\ Superconducting Super Collider Laboratory ${ }^{\ddagger}$ \\ 2550 Beckleymeade Avenue \\ Dallas, TX 75237 \\ and \\ M. Kummer \\ Department of Mathematics \\ University of Toledo \\ Toledo, OH 43606
}

October 1993

\footnotetext{
* Submitted for publication.

† On leave from Mathematics Department, University of New Mexico, Albuquerque, NM 87131.

$\ddagger$ Operated by the Universities Research Association, Inc., for the U.S. Department of Energy under Contract No. DE-AC35-89ER40486.
} 


\section{DISCLAIMER}

Portions of this document may be illegible in electronic image products. Images are produced from the best available original document. 


\title{
Theoretical Study of Longitudinal Beam Splitting and Related Phenomena
}

\author{
J. A. Ellison* and H.-J. Shih \\ Superconducting Super Collider Laboratory, \\ 2550 Beckleymeade Ave., Dallas, TX 75237 USA \\ M. Kummer \\ Department of Mathematics, University of Toledo, Toledo, $\mathrm{OH} 43606$ \\ (October 7, 1993)
}

\begin{abstract}
A recent experiment at the Indiana University Cyclotron Facility (IUCF) with electron cooling showed that if phase modulation near $1: 1$ resonance leads to longitudinal beam splitting. Here we explain this by applying the method of averaging, a powerful tool from the study of dynamical systems, to the underlying equation of motion-a pendulum equation with small damping and periodic forcing. The beam splitting is explained by showing that the associated Poincaré map has two attracting fixed points, each with a welldefined basin of attraction. Our approach can be immediately applied to other accelerator physics problems governed by a similar equation.
\end{abstract}

29.27.Bd, 41.85.Ct, 03.20.+i, 02.30.Hq

Typeset using REVTEX 
We begin by discussing the origin of the damped, forced pendulum equation in the context of longitudinal beam dynamics with rf phase modulation. We take the phase of the rf to be

$$
\phi_{\mathrm{rf}}(t)=\omega_{\mathrm{rf}} t+\psi(t),
$$

where $\omega_{\mathrm{rf}}$ is the $\mathrm{rf}$ frequency and $\psi(t)$ is the phase modulation. Let $t_{1}, t_{2}, \ldots$ be the times a particle is in the rf and $\phi_{n}=\phi_{\mathrm{rf}}\left(t_{n}\right)-\phi_{s, n}$ so that $\phi_{n}$ is the phase relative to the synchronous phase, $\phi_{s, n}$. Note that $\phi_{s, n+1}-\phi_{s, n}=\omega_{\mathrm{rf}} T_{0}=2 \pi h$, where $T_{0}$ is the revolution frequency and $h$ is the harmonic number. In the presence of damping, for example, due to electron cooling of proton beams at the Indiana University Cyclotron Facility (IUCF), the turn-toturn variations of the longitudinal phase space coordinates $(\phi, \delta)$ are [1]

$$
\begin{aligned}
& \phi_{n+1}=\phi_{n}+2 \pi h \eta \delta_{n+1}+\psi\left(t_{n+1}\right)-\psi\left(t_{n}\right) \\
& \delta_{n+1}=\delta_{n}+\frac{e V}{p v}\left(\sin \left(\phi_{n}+\phi_{s, n}\right)-\sin \phi_{s, n}\right)-\lambda \delta_{n}
\end{aligned}
$$

which in the continuous time approximation gives

$$
\ddot{\phi}+2 \alpha \dot{\phi}+K_{0}\left(\sin \left(\phi+\phi_{s}\right)-\sin \phi_{s}\right)=2 \alpha \dot{\psi}+\ddot{\psi}
$$

Here $K_{0}=-\left(2 \pi h \eta / T_{0}^{2}\right)(e V / p v), \eta$ is the slip factor, $\delta$ the relative momentum deviation, $e V$ the peak rf voltage, $p$ the synchronous momentum, $v$ the synchronous velocity and $\alpha=\lambda / 2 T_{0}$ a damping coefficient. This equation with $\alpha=0$ is important in understanding the effect of rf phase modulation on the Superconducting Super Collider (SSC) beam as well as in understanding proposals for crystal extraction using rf phase modulation [2]. The equation also arises in a completely different manner in the study of particle beam motion near a nonlinear betatron resonance in the presence of tune modulation, which was investigated in a Fermilab experiment, E778 [3]. In addition, it is the basic equation used for discussion of a recent experiment at the IUCF [4]. Here we focus on the IUCF experiment and use its parameters in our numerical calculations, but our results and analysis are also relevant to the other situations. 
We will consider only the storage mode so that $\phi_{s}=0$ or $\pi$. In the Indiana experiment $\psi(t)=F \frac{\omega_{s}}{\omega_{m}} \sin \left(\omega_{m} t+\Theta\right)$, where $\omega_{s}^{2}=\left|K_{0}\right|$ and $F$ is $\hat{B}_{m}$, dipole modulation amplitude, dependent but $\omega_{m}$ independent. Using this and taking $\tau=\omega_{m} t+\Theta$ as the independent variable, we obtain the initial value problem (IVP):

$$
\phi^{\prime \prime}+2 \frac{\alpha}{\omega_{m}} \phi^{\prime}+\frac{\omega_{s}^{2}}{\omega_{m}^{2}} \sin \phi=F \frac{\omega_{s}}{\omega_{m}}\left(2 \frac{\alpha}{\omega_{m}} \cos \tau-\sin \tau\right)
$$

with $\phi\left(\tau_{0}\right)=\phi_{0}$ and $\phi^{\prime}\left(\tau_{0}\right)=\phi_{0}^{\prime}$. The $2 \pi$-Poincare map (PM) in the $\tau=\tau_{0}$ section is obtained by integrating the IVP (4) from $\tau=\tau_{0}$ to $\tau=\tau_{0}+2 \pi$. All integrations of IVPs in this letter were done using RKF45 [5] with RELERR $=10^{-5}$ and ABSERR $=0$. In Fig. 1 we show the evolution of 50 points uniformly distributed on the negative $\phi$-axis in the $\tau_{0}=-\pi / 2$ section for $\omega_{m}=2 \pi(240) \mathrm{sec}^{-1}, \omega_{s}=2 \pi(262) \mathrm{sec}^{-1}, \alpha=2.5 \mathrm{sec}^{-1}$, and $F=0.0195$ (corresponding to $\hat{B}_{m}=4 \mathrm{G}$ ). The iterates of these points eventually settle down to two apparent fixed points of the PM: $\left(\phi_{e}, \phi_{e}^{\prime}\right)=(-1.1957,0.2146)$ and $(0.1119,0.0023)$. We have found these stable FPs of the PM by following two orbits until they settle down. It appears that each point in the region shown limits on one of the two fixed points; however, the basins of attraction are not clear, as nearby points can go to different attractors. Our goal is to explain this using the method of averaging [6], a powerful dynamical systems tool, which will interpret the complicated dynamics of (4) in terms of an autonomous system in the plane. The autonomous system in the plane is easily understood in terms of its phaseplane portrait. This then will allow us to characterize the basins of attraction and explain some of the features of the Indiana experiment, including the beam splitting.

To put (4) in a form for the method of averaging we need to introduce scaling and transformations. From Fig. 1 it appears that the effects of nonlinearity, forcing, and damping are all important. If there were no forcing, (4) would be a damped pendulum, and there would be only one fixed point (FP) at the origin in Fig. 1, and it would be a stable spiral point. (The FPs at $( \pm \pi, 0)$ are not in this region.) If nonlinearity were not important we could replace $\sin \theta$ by $\theta$, but then again there would be only one FP, a stable spiral. The fact that there appear to be asymptotically stable FPs shows that the damping is significant. 
We will treat all three effects as perturbations and introduce a small parameter $\epsilon$ in a way that brings in each effect at the same order in (4): $\phi=\epsilon^{1 / 2} \varphi_{1}, F=\epsilon^{3 / 2} \hat{F}$, and $\alpha / \omega_{s}=\epsilon \hat{\alpha}$. Here we assume $\varphi_{1}, \hat{F}$, and $\hat{\alpha}$ to be $O(1)$. Also because $\omega_{m}$ and $\omega_{s}$ are close we expect a $1: 1$ resonance, and so we take $\omega_{m} / \omega_{s}=: 1+\beta=: 1+\epsilon \hat{\beta}$, which yields

$$
\begin{aligned}
& \varphi_{1}^{\prime}=: \varphi_{2} \\
& \varphi_{2}^{\prime}=-\varphi_{1}+\epsilon g_{2}(\varphi, \tau)+\epsilon^{2} h_{2}(\varphi, \tau, \epsilon),
\end{aligned}
$$

where $g_{2}(\varphi, \tau)=+2 \hat{\beta} \varphi_{1}+\frac{1}{6} \varphi_{1}^{3}-2 \hat{\alpha} \varphi_{2}-\hat{F} \sin \tau$ and $h_{2}(\varphi, \tau, \epsilon)=O(1)$, i.e., $h_{2}$ is bounded as $\epsilon \rightarrow 0$. With $h_{2}=0$, this is just the Duffing equation with small damping, forcing, and nonlinearity near resonance, an equation which has been extensively studied in the dynamical systems literature (see Refs. 6(a), 6(b) and 6(d)). If we use the parameters of Fig. 1 with $\hat{F}=1$, then $\epsilon=0.07239, \hat{\alpha}=0.02098, \beta=-0.0840$, and $\hat{\beta}=-1.1600$.

Regular perturbation theory gives the $O(1)$ periodic solutions of (5) as $\varphi_{1}=r \cos (\tau-$ $\chi)+O(\epsilon)$ where

$$
\begin{aligned}
& \hat{\alpha} r-\frac{1}{2} \hat{F} \cos \chi=0 \\
& \hat{\beta} r+\frac{1}{16} r^{3}-\frac{1}{2} \hat{F} \sin \chi=0
\end{aligned}
$$

which follows from the expansion $\varphi_{1}=y_{0}+\epsilon y_{1}+O\left(\epsilon^{2}\right)$ and the removal of secular terms from the $y_{1}$ equation since $y_{1}$ must be periodic. In the PM, periodic solutions are fixed points. Solving (6) for the above parameters gives, in the coordinates of the PM of Fig. 1,

$$
\left(\begin{array}{l}
\phi_{e} \\
\phi_{e}^{\prime}
\end{array}\right)=\left(\begin{array}{c}
-1.1905 \\
0.2292
\end{array}\right),\left(\begin{array}{l}
0.1171 \\
0.0021
\end{array}\right),\left(\begin{array}{l}
1.0809 \\
0.1877
\end{array}\right) .
$$

Two correspond very well with the two apparent asymptotically stable FPs of Fig. 1, and the other, we shall see later, corresponds to an unstable FP of the PM. In Fig. 2(a) we show the amplitude $\Phi_{e}=\epsilon^{1 / 2} r$ of the periodic orbits as a function of $\beta$. There are three solutions for $\beta<\beta_{c} \simeq-0.04$ and one solution for $\beta>\beta_{c}$. The stable FPs of the PM, determined as above, are shown by the open circles. The agreement between the exact from (4) and the approximate from (6) is excellent for $\beta$ small. The solutions of Eq. (4) in Reference 4(b) are 
also shown (by crosses), and they also agree quite well for $\beta$ small. In Fig. 2 (b) we show the amplitude as a function of $\alpha$. We see that for large $\alpha$ the two large-amplitude periodic orbits coalesce and disappear. In Fig. 2(c) we show for fixed $F$ the regions in $(\alpha, \beta)$ space of 3 (shown by the shading) and 1 solutions. A scaling argument makes this universal (for arbitrary $F$ ).

We now proceed, using the method of averaging, to find an approximation to the PM for (4). The initial value problem for (5) can be written in vector form $\varphi^{\prime}=J \varphi+\epsilon g(\varphi, \tau)+$ $\epsilon^{2} h(\varphi, \tau, \epsilon), \varphi\left(\tau_{0}, \tau_{0}, \xi, \epsilon\right)=\xi$, where $\varphi=\varphi\left(\tau, \tau_{0}, \xi, \epsilon\right), J=(0,1 ;-1,0), \varphi=\left(\varphi_{1}, \varphi_{2}\right)^{T}$ $g=\left(0, g_{2}\right)^{T}$, and $h=\left(0, h_{2}\right)^{T}$. Defining $x$ by the variation-of-parameters transformation,

$$
\varphi=e^{J\left(\tau-\tau_{0}\right)} x
$$

yields the IVP for $x=x\left(\tau, \tau_{0}, \xi, \epsilon\right)$ :

$$
\begin{gathered}
x^{\prime}=\epsilon f\left(x, \tau, \tau_{0}\right)+\epsilon^{2} R, \quad x\left(\tau_{0}, \tau_{0}, \xi, \epsilon\right)=\xi, \\
f\left(x, \tau, \tau_{0}\right)=e^{-J\left(\tau-\tau_{0}\right)} g\left(e^{J\left(\tau-\tau_{0}\right)} x, \tau\right),
\end{gathered}
$$

and $R=R\left(x, \tau, \tau_{0}, \epsilon\right)$ defined analogously. We have introduced $\tau_{0}$ in (7) so that in the $\tau_{0}$ section $\varphi=x$. This problem is now in a standard form for the method of averaging. The averaged problem is

$$
\begin{gathered}
u^{\prime}=\epsilon \bar{f}\left(u, \tau_{0}\right), \quad u\left(\tau_{0}, \tau_{0}, \xi, \epsilon\right)=\xi, \\
\bar{f}\left(u, \tau_{0}\right)=\frac{1}{2 \pi} \int_{0}^{2 \pi} f\left(u, \tau, \tau_{0}\right) d \tau \\
=\left(\begin{array}{c}
-\hat{\alpha} u_{1}-\hat{\beta} u_{2}-\frac{1}{16}\left(u_{1}^{2}+u_{2}^{2}\right) u_{2}+\frac{1}{2} \hat{F} \cos \tau_{0} \\
-\hat{\alpha} u_{2}+\hat{\beta} u_{1}+\frac{1}{16}\left(u_{1}^{2}+u_{2}^{2}\right) u_{1}-\frac{1}{2} \hat{F} \sin \tau_{0}
\end{array}\right) .
\end{gathered}
$$

If we define $v=v\left(s, \xi, \tau_{0}\right)$ by $d v / d s=\bar{f}\left(v, \tau_{0}\right), v\left(0, \xi, \tau_{0}\right)=\xi$, then $u\left(\tau, \tau_{0}, \xi, \epsilon\right)=$ $v\left(\epsilon\left(\tau-\tau_{0}\right), \xi, \tau_{0}\right)$, and a version of the first-order averaging theorem gives

$$
x=u+\epsilon P\left(u, \tau, \tau_{0}\right)+O\left(\epsilon^{2}+\epsilon^{2}\left(\tau-\tau_{0}\right)\right)
$$


for $0 \leq \tau-\tau_{0} \leq O(1 / \epsilon)$. Here $x$ and $u$ have the same arguments and $P\left(u, \tau, \tau_{0}\right)=$ $\int_{\tau_{0}}^{\tau}\left(f\left(u, s, \tau_{0}\right)-\bar{f}\left(u, \tau_{0}\right)\right) d s$. It follows that

$$
\varphi\left(\tau_{0}+2 \pi n, \tau_{0}, \xi, \epsilon\right)=u\left(\tau_{0}+2 \pi n, \tau_{0}, \xi, \epsilon\right)+O\left(\epsilon^{2} n\right)
$$

and thus the geometry of the Poincaré sections for (5) (and (4)) will be close to the phaseplane portraits for (10) for $\epsilon$ sufficiently small.

The equilibrium solutions of the averaged problem, $u=u_{e}$, are precisely those given by regular perturbation theory discussed earlier. To see this, let $u_{e 1}=r \cos \theta$ and $u_{e 2}=r \sin \theta$; then $\bar{f}\left(u_{e}, \tau_{0}\right)=0$ gives $(6)$, with $\chi=\tau_{0}+\theta$ (and (7), with $x$ replaced by $u_{e}$, gives the periodic solutions obtained from regular perturbation theory). Linearizing about the equilibrium solutions and solving the associated eigenvalue problems gives the eigenvalues

$$
\left(\begin{array}{l}
\lambda_{1} \\
\lambda_{2}
\end{array}\right)=\left(\begin{array}{c}
a+i b \\
a-i b
\end{array}\right),\left(\begin{array}{c}
a+i c \\
a-i c
\end{array}\right),\left(\begin{array}{c}
-0.0369 \\
0.0335
\end{array}\right)
$$

where $a=-0.0017, b=0.0389$, and $c=0.0822$. Thus the linearization has two asymptotically stable spirals and a saddle point. Since the equilibria are hyperbolic, (10) has the same structure as the linearization near them. This is illustrated in Fig. 3, where we show the phase-plane portrait for $\tau_{0}=-\pi / 2$. The four non-constant trajectories shown are the stable and unstable manifolds of the saddle point. The manifolds were computed numerically, using initial conditions near the saddle point on the eigenvectors: $(-0.4411,1.0)$ and $(0.0717,1.0)$. The basins of attraction associated with the two attractors (one is shown by the shading) are clearly defined by the stable manifolds. The evolution for an arbitrary initial condition is now easily inferred.

The averaging theorem says the phase-plane portrait of $(10)$ and the Poincare section of (5) will be close for $\epsilon$ small (and $n$ not too large). However, we can say more about the original problem in terms of the PM: $\mathcal{P}_{\tau_{0}}(\xi, \epsilon):=\varphi\left(\tau_{0}+2 \pi, \tau_{0}, \xi, \epsilon\right)=v\left(2 \pi \epsilon, \xi, \tau_{0}\right)+O\left(\epsilon^{2}\right)$. For $\epsilon$ sufficiently small, an application of the implicit function theorem shows that the PM has fixed points $O\left(\epsilon^{2}\right)$ close to the equilibrium solutions of the averaged problem (and, of course, FPs of the PM are periodic solutions of (5)). Indeed, this is illustrated in Fig. 2a, as 
we have discussed. Since the equilibrium solutions of (10) are hyperbolic, Theorem 4.1.1 in Reference 6(b) asserts that for $\epsilon$ small the phase-plane structure persists in the PM (other good references for this material are $6(\mathrm{a}), 6(\mathrm{~d})$ and $6(\mathrm{e}))$. Thus we have explained the dynamics of (4), including Poincaré surface of section results in Fig. 1, in terms of the phaseplane portrait in Fig. 3, and this in turn explains the beam splitting in the IUCF experiment in terms of the two attractors of the averaged system and their basins of attraction. We have taken $\tau_{0}=-\pi / 2$ in Figs. 1 and 3, but clearly the beam splitting should not depend on a particular surface of section. In fact, the phase-plane portraits of (10) for different values of $\tau_{0}$ are obtained simply by rotation, since the coordinate transformation $w=e^{J \gamma} u$, which is a rotation, gives $\dot{w}=\bar{f}\left(w, \tau_{0}+\gamma\right)$, as can be seen from (9).

Finally, we discuss the case $\alpha=0$ for $F$ of Fig. 1, which is relevant to the IUCF experiment as well as to the E778 experiment. In Fig. 4(a) we show the phase-plane portrait of the averaged system for $\beta=-0.0840$. This is similar to the result obtained in Ref. 4 (c) using formal canonical perturbation theory. The implicit function theorem can again be applied to show that the equilibrium solutions correspond to periodic solutions of (5). The persistence of the invariant circles for $\epsilon$ small is a deeper result that follows from the Moser Twist theorem if the frequency of the periodic solutions of the averaged system as a function of action has a non-zero derivative at zero action. We have verified that this is the case. Also there are two homoclinic orbits (dashed curves in Fig. $4(\mathrm{a})$ ), and these presumably do not persist in the PM. The stable and unstable manifolds most likely intersect transversely, with transcendentally small angle, giving rise to a thin stochastic layer. However, this is very difficult to prove, as the literature on the rapidly forced pendulum, a prototype problem, shows. (See, for example, Reference 7.)

As $\beta$ increases from its value in Fig. 4(a), a bifurcation to one equilibrium solution occurs at $\beta=\beta_{c}:=-3(\sqrt{2} F)^{2 / 3} / 8=-0.0342\left(\right.$ or $\omega_{m}^{2} / \omega_{s}^{2}=(1+\beta)^{2} \simeq 1-3(4 F)^{2 / 3} / 8$, corresponding to the formula of Reference 4(a)). Figure 4(b) shows the phase-plane portrait for $\beta=\beta_{c}$ and indicates a cusp structure at the bifurcation point. Figure $4(\mathrm{c})$ shows the on-resonance case $(\beta=0)$, and all solutions are periodic. Again the Moser Twist theorem 
can be applied to determine stability of the associated FP of the PM. This bifurcation is interesting because the two stable solutions are quite separated in phase space, and so the bifurcation could perhaps be observed in the IUCF experiment.

This work has been supported by the Superconducting Super Collider Laboratory, which is operated by the Universities Research Association, Inc., for the U.S. Department of Energy under contract No. DE-AC35-89ER40486. Discussions with S.Y. Lee, M.J. Syphers, and Y.T. Yan are gratefully acknowledged. 


\section{REFERENCES}

* On leave from Mathematics Department, University of New Mexico, Albuquerque, NM 87131.

[1] G. Dôme, "Diffusion Due to RF Noise," in CERN Advanced Accelerator School on Advanced Accelerator Physics, CERN Report No. 87-03, 1987, pp. 320-401.

[2] H.-J. Shih, J. A. Ellison, B. S. Newberger, and R. Cogburn, "Longitudinal Beam Dynamics with RF Noise," to be published in Particle Accelerators.

[3] (a) T. Satogata et al., Phys. Rev. Lett. 68, 1838 (1992); (b) T. Chen, S. Peggs, and G. Tsironis, in Proceedings of the Second European Particle Accelerator Conference (Editions Frontières, Gif-sur-Yvette, France, 1990), Vol. 2, pp. 1753-1755.

[4] (a) M. Ellison et al., Phys. Rev. Lett. 70, 591 (1993); (b) M. Syphers et al., Phys. Rev. Lett. 71, 719 (1993); (c) H. Huang et al., preprint.

[5] RKF45 is a variable-step Runge Kutta method discussed, for example, in G.E. Forsythe, M.A. Malcolm, and C.B. Moler, Computer Methods for Mathematical Computations (Prentice-Hall, New Jersey, 1977).

[6] There are many good references to the method of averaging, here we mention several relevant to this paper: (a) J. K. Hale, Ordinary Differential Equations (Krieger, Florida, 1980); (b) J. Guckenheimer and P. Holmes, Nonlinear Oscillations, Dynamical Systems and Bifurcations of Vector Fields, (Springer-Verlag, New York, 1986); (c) H. S. Dumas, J. A. Ellison, and A. W. Sánez, Annals of Phys. 209, 97 (1991); (d) J. A. Murdock, "Perturbations: Theory and Methods," (Wiley, New York, 1991); (e) J. Murdock, "Qualitative Theory of Nonlinear Resonance by Averaging and Dynamical Systems Methods," in Dynamics Reported, Vol. 1 (U. Kirchgraber and H. O. Walther, eds.), Wiley, New York, 1988. 
[7] J. A. Ellison, M. Kummer, and A. W. Sáenz, J. of Dynamics and Differential Equations, April 1993. 


\section{FIGURES}

FIG. 1. Evolution of 50 points in the Poincare $\tau_{0}=-\pi / 2$ section of Eq. (4) indicating the existence of two attractors. The points are initially uniformly distributed between -2 and 0 . See text for the parameters used.

FIG. 2. (a) Amplitude of periodic solutions as a function of $\beta$, for $\alpha=2.5$, obtained from Eq. (6) (solid line). Circles correspond to amplitudes determined directly from Eq. (4); crosses are

discussed in the text. (b) Amplitude as a function of $\alpha$ for $\beta=-0.0840$. (c) Regions in $\alpha, \beta$ plane corresponding to one and three solutions. In (a), (b), and (c) $F=0.0195$.

FIG. 3. Phase-plane portrait of Eq. (10) for $\tau_{0}=-\pi / 2$. The parameters are the same as in Fig. 1.

FIG. 4. As in Fig. 3 for $\alpha=0$. (a) $\beta=-0.0840$. (b) $\beta=\beta_{c}=-0.0342$. (c) $\beta=0$. 


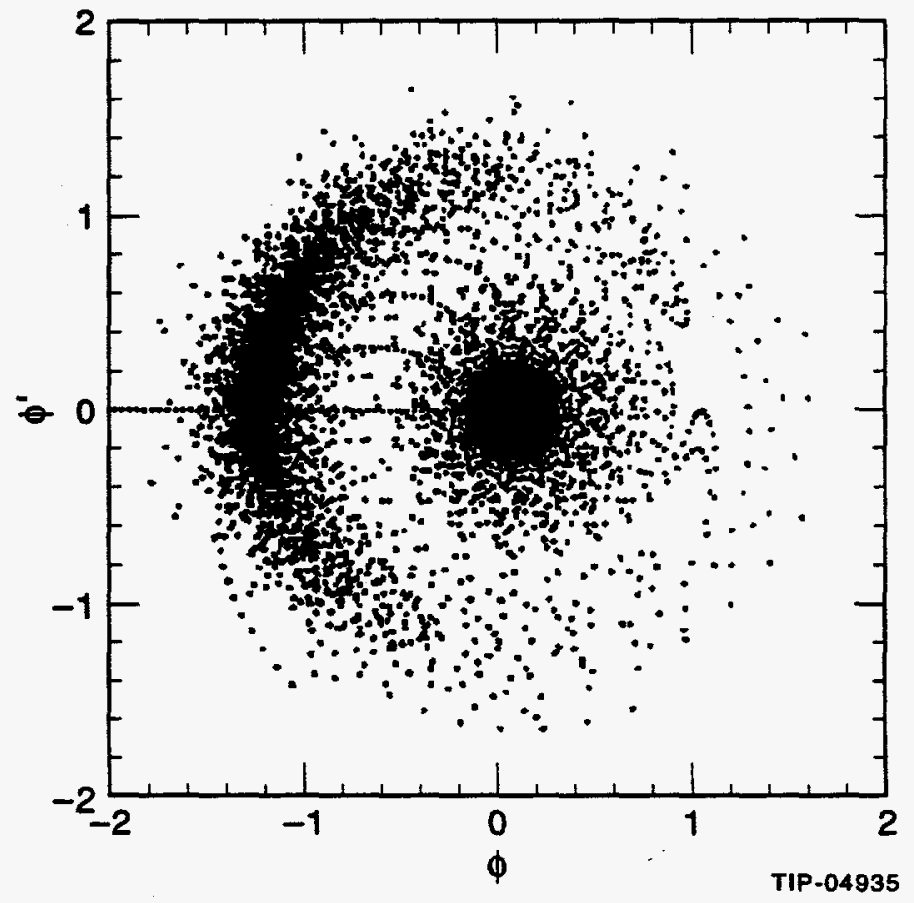

Figure 1 
(a)

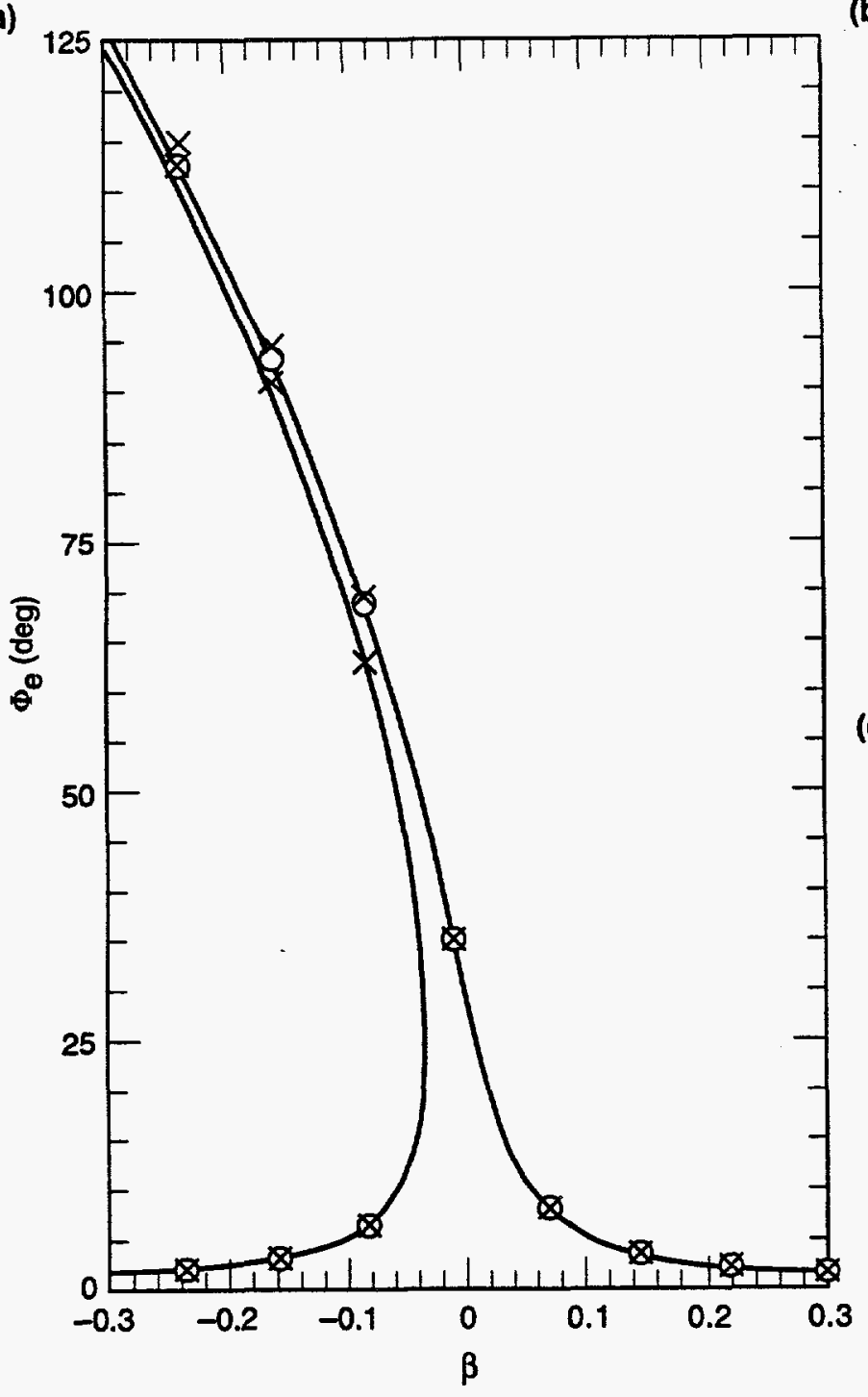

(b)

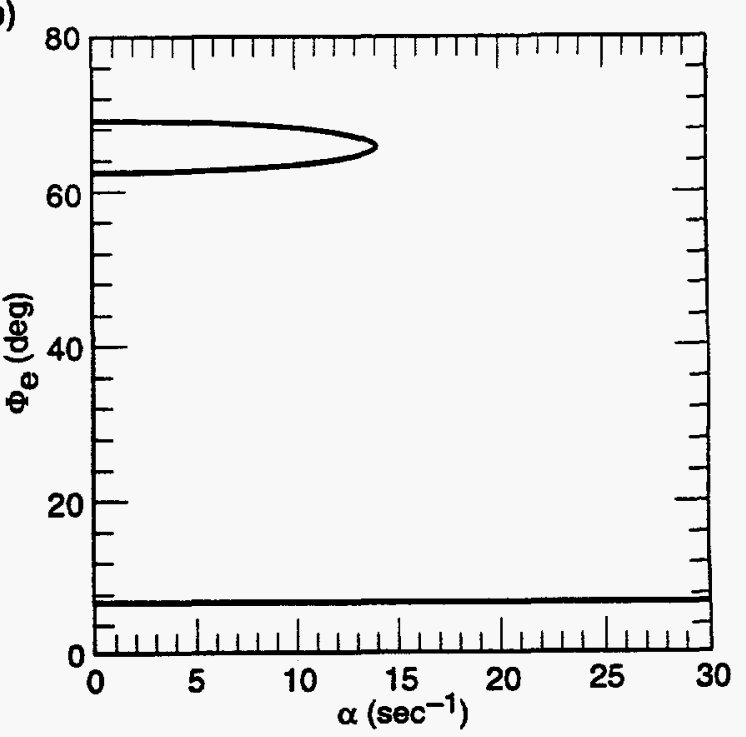

(c)

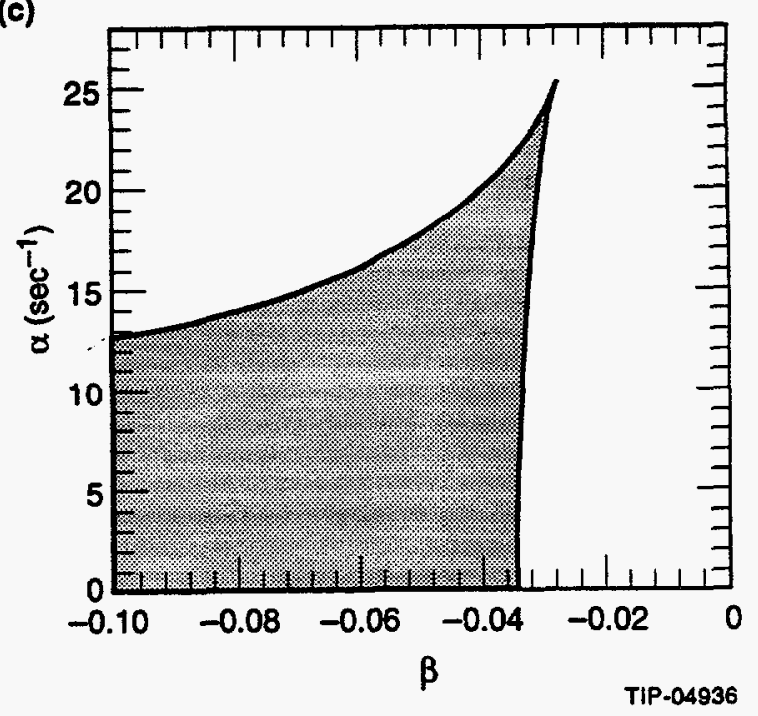




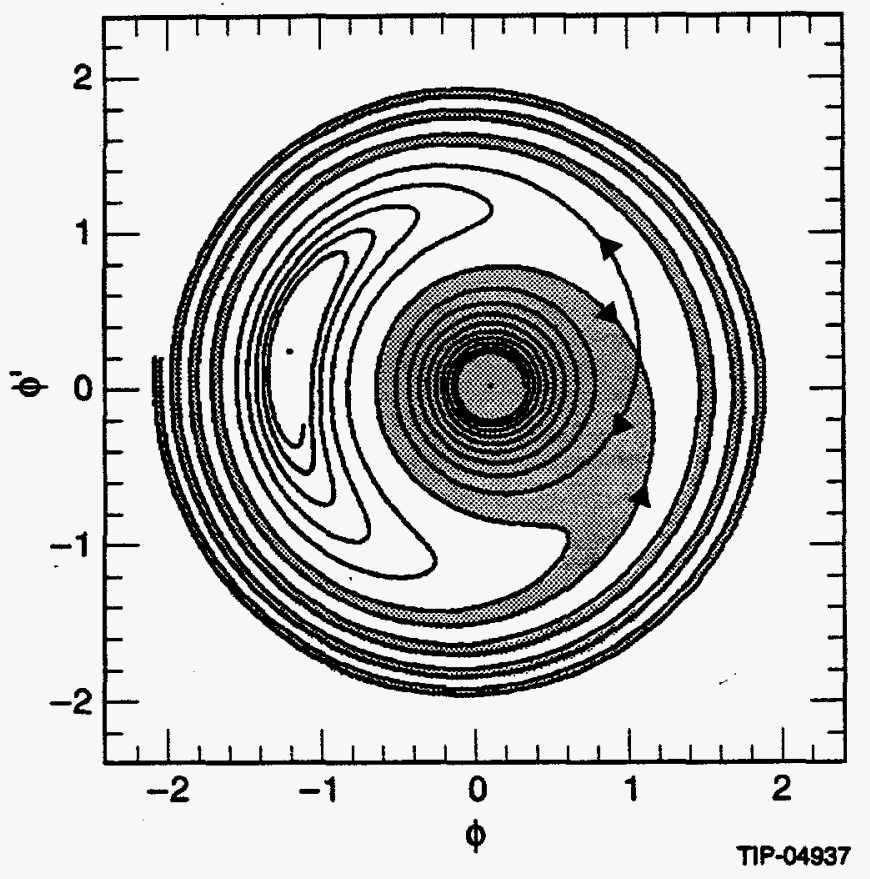

Figure 3 

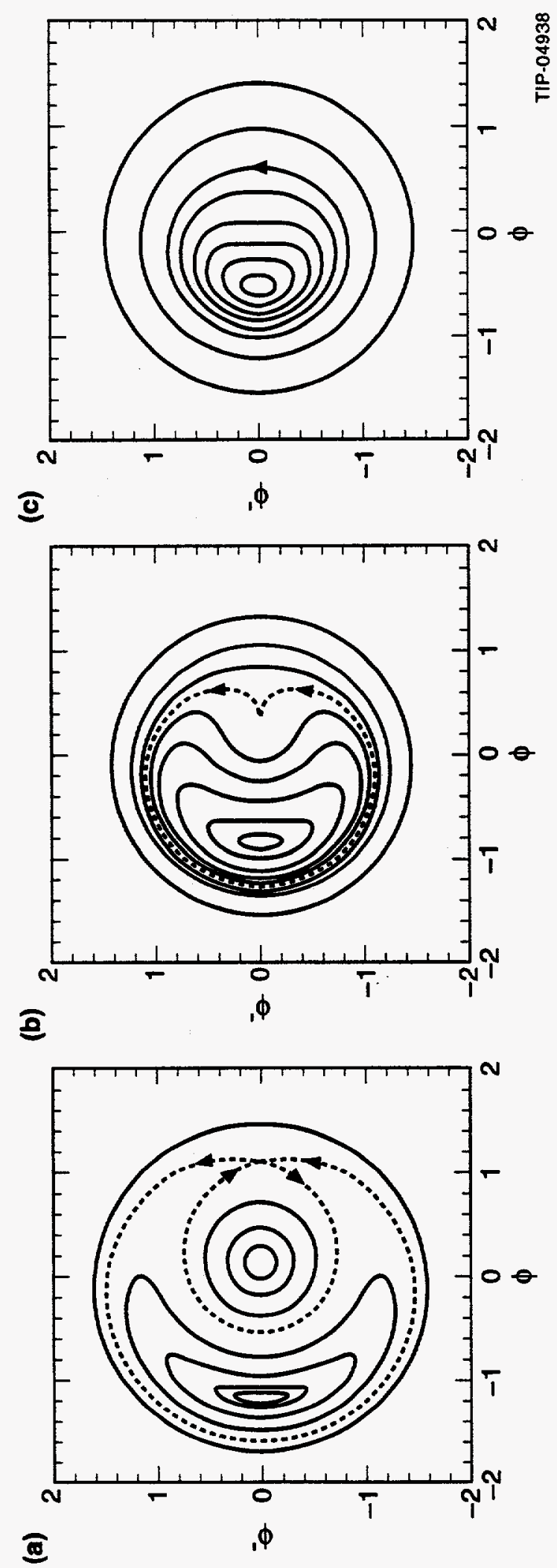